\title{
Are all cytokine storms the same?
}

\author{
Nicki Panoskaltsis ${ }^{1,2} \mathbb{E}_{0}$
}

Received: 31 August 2020 / Accepted: 7 December 2020 / Published online: 8 January 2021

(c) The Author(s), under exclusive licence to Springer-Verlag GmbH, DE part of Springer Nature 2021

\begin{abstract}
Cytokine release syndrome (CRS) is the result of massive pro-inflammatory cytokine release and imbalance in the absence of adequate immunomodulation from signals such as interleukin (IL)-10, resulting in ongoing inflammation, tissue damage and death if left uncontrolled. Although CRS can result from different pro-inflammatory insults, the treatments proposed are similar, regardless of the phase of response. SARS-CoV-2 causes COVID-19, and CRS has been a defining feature of severe disease. Common approaches to treating CRS in other conditions are now applied to COVID-19 and, although some patients respond, it begs the following questions: (1) are all cytokine storms the same regardless of initiating insult, (2) can treatments be considered equally for all CRS events at any phase of the response, (3) can CRS be predicted based on dynamic acute biomarkers and, (4) should patients with CRS undergo long-term monitoring for secondary effects? The aim of this commentary is not to provide a review of COVID-19 pathophysiology or of cytokine storm, but rather to establish a foundation which could act as a platform to inform treatment approaches to CRS, regardless of cause, and the short- and long-term follow-up which may be necessary for affected patients.
\end{abstract}

Keywords Cytokine storm · Cytokine release syndrome · CRS · TGN1412 · COVID-19 - SARS-CoV-2

Cytokine release syndrome (CRS) is the result of a massive pro-inflammatory cytokine release and imbalance in the absence of adequate immunomodulation from signals such as interleukin (IL)-10, resulting in ongoing inflammation, tissue damage and death if left uncontrolled [1-4]. It manifests clinically with high fever, delirium, nausea, vomiting, abdominal pain, diarrhea and, if untreated, can progress to hypotension and multi-organ failure often with acute hypoxemia due to an ill-defined acute lung injury [1-3]. Hematologically, disseminated intravascular coagulation and/or a pro-thrombotic coagulopathy often results, with thrombocytopenia and anemia; there is a discrete absence of T-cells and monocytes from the peripheral blood in the acute phase of the cytokine storm, followed by deliberate and dynamic recovery over time, which is cell-subset-dependent [1].

Nicki Panoskaltsis

nicki.panoskaltsis@emory.edu

1 Department of Hematology and Medical Oncology, Winship Cancer Institute, Emory University School of Medicine, Atlanta, GA 30322, USA

2 BioMedical Systems Engineering Laboratory, Wallace H. Coulter Department of Biomedical Engineering, Georgia Institute of Technology, Atlanta, USA
Neutrophils are spared and often increased, in contrast to the neutropenia observed in macrophage activation syndrome/hemophagocytic lymphohistiocytosis, distinguishing CRS from these related conditions [1,5]. Predicting which patient will have CRS, and anticipating the severity of illness, has been a challenge since cytokine storm is initiated in the target tissue(s), a site which is often unknown until damage has occurred [1-3, 6]. Peripheral blood biomarkers of CRS are used for diagnosis and to guide therapy even though they reflect what has already transpired and what remains after cell redistribution to tissues or cell death [2, $3,5,6]$. CRS is a dynamic process and what is observed on single-time-point blood sampling, may not be reflective of physiological processes requiring treatment $[1,7,8]$. Furthermore, target tissue(s) and cell(s) may differ depending on the instigating agent (e.g. pathogen vs CAR-T-cell targeting of tumors), route of entry into the host, and between individuals in response to the same agent (Fig. 1A) $[1-3,5,6]$. SARS-CoV-2 causes COVID-19 and CRS has been a defining feature of severe disease [5, 7-9]. Although the current focus has been on treating CRS induced by SARS-CoV-2, the approach assumes that all cytokine storms are the same thereby justifying similar treatment targets throughout the CRS course [2-4, 6, 9-11]. Targeting the action of one of the 


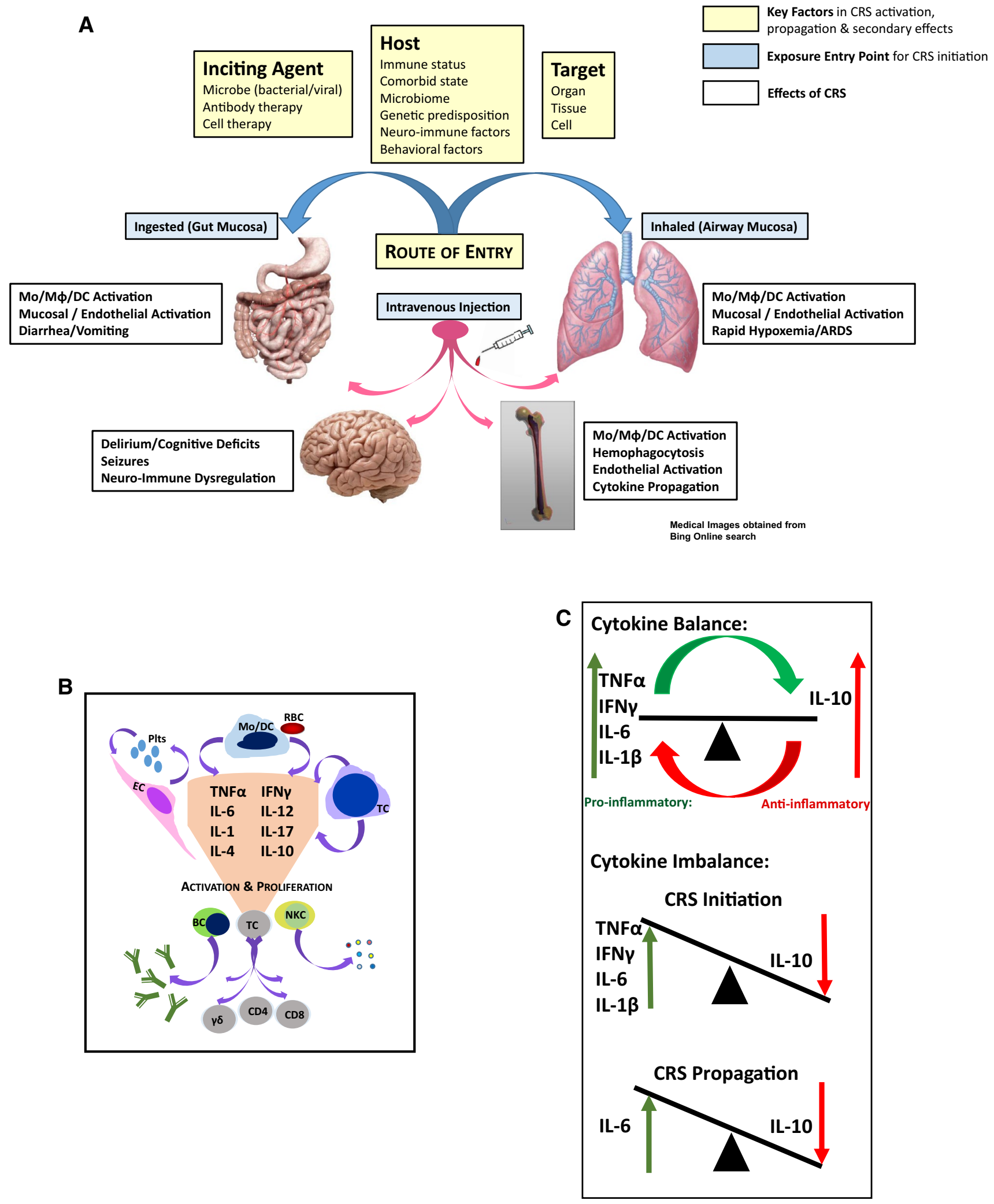


4 Fig. 1 Dynamics of immune imbalance in CRS. A Key factors in CRS activation, exposure entry points and on- or off-target effects of the inciting agent. Other organs not included here can also be affected, e.g. heart, liver and kidneys. B Activation of cells in the target tissues initiate a cytokine cascade and activation of other cell types resulting in cell proliferation, further cytokine release and propagation of tissue damage. C Cytokine balance usually ensues following innate immune activation with the inciting agent. Cytokine imbalance with a massive release of pro-inflammatory cytokines initiates CRS, which is further propagated by prolonged and excessive IL-6 levels or inadequate IL-10 expression. D The temporal dynamics of CRS shows an initial innate immune response with an early release of pre-stored TNF $\alpha$, followed immediately by IFN- $\gamma$, then IL- $1 \beta$, and IL-6. IL-10 is released in response to the pro-inflammatory cytokines to immunomodulate early on, but an inadequate counterbalancing level (downward arrow), or prolonged IL-1 $\beta$ or IL-6 levels (horizontal arrows) will cause increasing CRS toxicities, including multi-organ failure, neurotoxicity and death. Bone marrow and T-cell responses are shown over a one year period. The T-cell peak is much earlier in the case of CAR-T-cell infusion. The dynamics of T-cell subsets is varied with $\mathrm{CD}^{+} \mathrm{RO}^{+}$or $\mathrm{CD}^{+} \mathrm{RO}^{+}$cells (dependent on the initiating agent, e.g. targeted antibody vs virus) stimulated primarily over the first months, followed by a transition to an increase in effector memory T-cells. Tregs cycle circannually at low levels throughout and in those who have $\gamma \delta$ Tcell responses, an initial rise is followed by a gradual decline over years. Monocytes and DCs have different kinetic signatures, and RBCs follow the recovery trajectory of cDCs, possibly related to resolved hemophagocytosis following CRS. Dynamic treatment options, informed by the temporal stage of CRS and the immune imbalance which exists at the time, are shown, both in the immediate acute phase within the first 10 days, as well as in the follow-up phase during which immune- and organ-specific monitoring would inform treatment. Further potential cytokine release, at lower but more persistent levels, may occur in some patients concurrent with organ-specific symptoms (e.g. headache, gut irritability) following the release of CRS immunomodulation and recovery of immune cell subsets during the acute phase. CRS: Cytokine Release Syndrome; Mo: Monocyte; M $\varphi$ : Macrophage; DC: Dendritic Cell; ARDS: Acute Respiratory Distress Syndrome; RBC: Red Blood Cell; Plts: Platelets; EC: Endothelial Cell; TC: Tumor Cell; BC: B-Cell; Tc: T-cell; NKC: Natural Killer Cell; TNFo: Tumor Necrosis Factor alpha; IFN $\gamma$ : Interferon gamma; IL: Interleukin; cDC: Classical Dendritic Cell; pDC: Plasmacytoid Dendritic Cell; FU: Follow-up; ANTI: Antibody against specified cytokine or cytokine receptor action; JAK1/2i: Janus Kinase 1 or 2 inhibitor; BTKi: Bruton Tyrosine Kinase inhibitor; mTORi: mammalian Target of Rapamycin inhibitor

pathogenic cytokines in CRS, IL-6, with Tocilizumab and the remainder of the CRS with steroids are common firstand second-line therapies for cytokine storm induced by immunotherapeutics such as bispecific antibodies or CAR-T cells in leukemia and lymphoma [2]. For most patients in this clinical context, the treatment is highly effective and often completely reverses the toxicities of CRS. However, data have thus far not supported the use of Tocilizumab for CRS in COVID-19 [12]. Moreover, consistent monitoring of patients for secondary effects of CRS (and of the treatment given) is lacking. These fundamental principles beg the following questions: (1) are all cytokine storms the same regardless of initiating insult, (2) can treatments be considered equally for all CRS events at any phase of the response,
(3) can CRS be predicted based on dynamic acute biomarkers and, (4) should patients with CRS undergo long-term monitoring?

\section{Are all cytokine storms the same?}

The focus of CRS, and treatment for it, has been on the proinflammatory cytokines released in large amounts that can be detected at single time-points of the response-mainly IL-6 and IL-1 $\beta$ - or on cells thought to be central to the CRS, mainly monocytes, dendritic cells (DCs) and endothelial cells (ECs), likely of the target tissues, or tumor cells in the event of therapeutically-induced CRS (Fig. 1B) [2-4, 6, 9-11]. Although it is clear that cytokines released from tissue monocytes and DCs are critical in the propagated CRS response, the initiating event starts with the near-immediate release of $\mathrm{TNF} \alpha$ and IFN $\gamma$ which are likely no longer detectable by the time patients present [1-3]. Furthermore, the release of these cytokines and effects on target cells, such as bone marrow monocytes and DCs, initiate a cascade of events prompting the hemophagocytic response that, in itself, is immunomodulating via the release of IL-10 [13]. The timing of when a patient presents with CRS will dictate the cytokines and cell subsets identified and the phase of response targeted for treatment. With a naturally-occurring stimulus (pathogen), diagnosis will likely be later in the CRS cascade targeting IL-6/IL-10 imbalance. In contrast, for an artificial stimulus or therapeutic, such as CAR-T-cells, patients are monitored during treatment, and elevated TNF $\alpha$, IFN $\gamma$, IL-1 $\beta$ or IL-6 levels in balance with IL-10 may be detected, depending on the target cell and tumor burden (Fig. 1C) [1-3].

\section{Can all treatments be considered equally for any CRS event at any phase of the response?}

To answer this question, one must (a) address the cytokine imbalance caused by CRS leading to tissue damage, (b) target the treatment to the site of CRS action and, (c) mitigate potential off-target systemic effects with anti-CRS therapy. IL-10 seems to be crucial for tempering CRS and supplementation of this cytokine to restore balance may be a preferable option to blocking pro-inflammatory cytokines (IL-6, TNF $\alpha$ or IL-1 $\beta$ ), which could exacerbate infectious pathology $[1,13]$. Monocytes and DCs phagocytose erythrocytes and apoptotic bodies during acute inflammation/CRS leading to CRS-associated anemia and IL-10 release [1, 13]. Blood transfusions are immunomodulatory; targeting marrow monocyte/DC populations with transfusions, apoptotic bodies or other nanoparticle (possibly with cytokine loads for directed delivery) may 


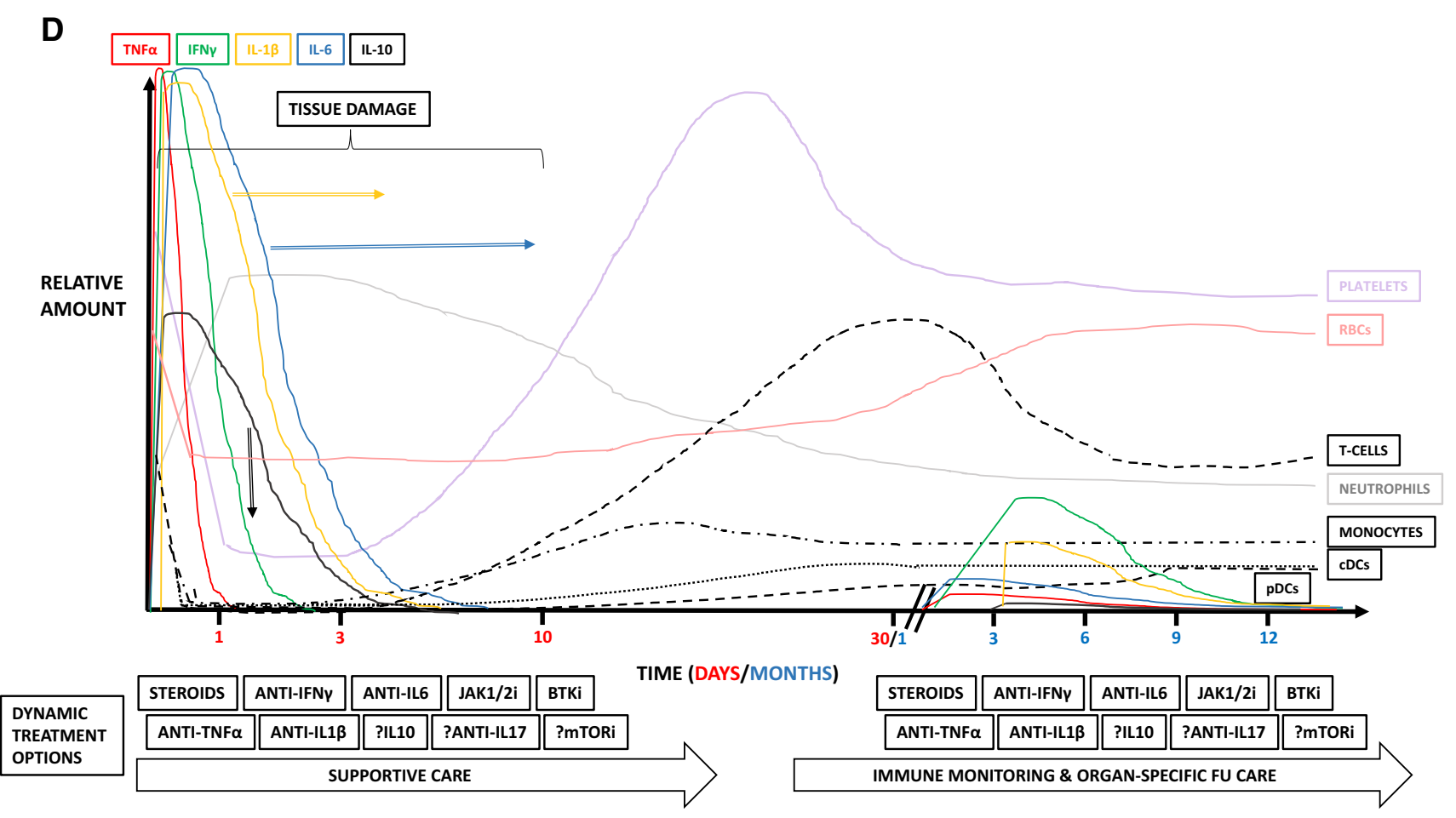

Fig. 1 (continued)

have similar yet more effective results, aimed at the site of CRS instigation and propagation. Targeting intracellular pathways such as Bruton Tyrosine Kinase (BTK) or Janus Kinase (JAK) may also achieve immune equipoise, though long-term or off-target effects of such treatments in the setting of CRS are currently unclear (Fig. 1D) [9]. One of the main challenges in achieving appropriate balance during CRS treatment is to reduce the toxicity induce by cytokine storm yet, in so doing, not sacrifice effective treatment of the underlying pathology, whether it be pathogen or tumor. For example, the treatment of severe CRS with Tocilizumab during CAR-T cell therapy, in general, is not significantly compromised since the tumor target has already been, and continues to be, engaged. In contrast, for COVID-19, antiviral immune responses which include those cytokines implicated in cytokine storm, are important for the eradication of SARS-CoV-2. The timing of anti-CRS therapy will therefore be critical such that it does not adversely impact antiviral immunity. Hence, short-term improvements in morbidity due to CRS treatment ultimately may not improve mortality in patient subsets if the timing of treatment and context of the disease are not adequately considered.

\section{Can CRS be predicted based on dynamic acute biomarkers?}

Observations made during the unfortunate TGN1412 clinical trial have provided human data of CRS pathophysiology in the absence of pathogen, contaminant or host comorbidities [1]. Six previously healthy young men were infused with the anti-CD28 superagonist, intended to induce a T-regulatory immune response. In contrast, all six suffered from a life-threatening CRS, initiated by $\mathrm{TNF} \alpha$ - already at maximum levels within an hour of infusion-followed by lymphopenia with monocytopenia and rapid-onset hypoxemia requiring critical care support, similar to the effects induced in COVID-19. Although the immunological basis for CRS has remained unclear, it was likely stimulated via innate immune populations or ECs of the lung, gut or bone marrow given the rapid release of TNF $\alpha$ [1]. One patient had a reduced IL-10 response and prolonged critical illness in addition to an ill-defined peripheral vascular syndrome resulting in dry gangrene, suggesting that the cytokine imbalance, possibly due to impaired hemophagocytosis since his anemia was mild, was the main factor in the severity of his illness [1, 14]. All patients recovered monocytes by day 10 , yet classical and plasmacytoid DCs took longer to recover indicative perhaps of more involved roles in CRS [1, 14]. T-cells slowly recovered with specific subset dynamics from day 
3 when cytokine trajectories declined, with circannual cycling thereafter (e.g. Tregs), indicative of the inferiority of single time-point samples for prediction and in directing therapeutic strategy (Fig. 1D) $[1,15,16]$. Though CRP and IL-6 levels have been helpful in diagnosing CRS in patients receiving CAR-T-cells, they often fail to accurately capture the severity of CRS and response to therapy $[2,3,6]$. Assessing IL-10 potential in monocytes/DCs may be a better predictor of CRS imbalance and risk of morbidity/mortality.

\section{Should patients with CRS undergo long-term monitoring?}

Although all patients who received TGN1412 survived due to aggressive supportive care, the cohort had ongoing cognitive, psychological, gastrointestinal, integumentary, and immune regulatory differences that persisted for years following the event [14-16]. If these are the effects in young, healthy individuals, the long-term effects in comorbid patients may be compounded by comparison, yet concerted efforts to qualify or quantify this CRS aftermath are lacking. It is probable, that the clinical symptoms and immunological signs observed in the short- and long-term following CRS will be a reflection of the main target organ(s) affected by the initiating event and route of entry.

So, are all CRSs the same and can they be treated similarly? Given that the features underlying CRS share a common cytokine imbalance the answer is yes-and no. Treatment for CRS should be commensurate with common features but also address differences in tissue and cell targets, and most importantly, the response dynamics in the individual patient. Rather than treating the main proinflammatory cytokines-IL-6, IL-1 $\beta$ or TNF $\alpha$ - treatment should dynamically focus on restoring balance. In contrast to the otherwise healthy young cohort infused with TGN1412, immune response dynamics in comorbid patients who are unwell due to a pathogen can be difficult to discern. Moving forward, a platform for CRS diagnosis, monitoring and treatment could be developed by applying the following considerations: (a) inciting agent, (b) route of exposure, (c) target and responder cell(s), (d) cytokine release and balance, (e) time-course of CRS and, (f) long-term follow-up for secondary effects of CRS (Fig. 1D). With these considerations, and the institution of rapid immune-profiling systems to account for CRS cell subset and cytokine biomarkers, goals of restoring immune balance may be achieved without risking imbalance in other areas of the pathogen response or increased long-term immune effects of therapy, which are currently unclear.
Funding None.

\section{Compliance with ethical standards}

Conflict of interest The author declares no potential conflicts of interest.

Statement of translational relevance Cytokine storm results from massive pro-inflammatory cytokine release and imbalance and can cause severe symptoms or death following cancer immunotherapy or infection. It is unclear if all cytokine storms are the same regardless of cause, justifying similar treatments, or even if biomarkers for severity and long-term effects can be established. Cytokine release syndrome has been a defining feature of severe COVID-19 and treatments designed to target this aspect of the disease are currently a high priority. Herein, a clinically-relevant dynamic approach to cytokine storm is proposed for treatment and follow-up in affected patients. Consideration is given to potential short- and long-term secondary effects of cytokine release syndrome which may be anticipated based on experience and longterm observations in a previously healthy cohort who suffered from TGN1412-induced cytokine storm.

\section{References}

1. Suntharalingam G, Perry M, Ward S, Brett SJ, Castello-Cortes A, Brunner MD et al (2006) Cytokine storm in a phase 1 trial of the anti-CD28 monoclonal antibody TGN1412. N Engl J Med 355:1018-1028

2. Teachey DT, Lacey SF, Shaw PA, Melenhorst JJ, Maude SL, Frey $\mathrm{N}$ et al (2016) Identification of predictive biomarkers for cytokine release syndrome after chimeric antigen receptor T-cell therapy for acute lymphoblastic leukemia. Cancer Discov 6:664-679

3. Hay KA, Hanafi L-A, Li D, Gust J, Liles WC, Wurfel MM et al (2017) Kinetics and biomarkers of severe cytokine release syndrome after CD19 chimeric antigen receptor-modified T-cell therapy. Blood 130:2295-2306

4. Liu Y, Fang Y, Chen X, Wang Z, Liang X, Zhang T et al (2020) Gasdermin E-mediated target cell pyroptosis by CAR T cells triggers cytokine release syndrome. Sci Immunol. 5:eaax7969

5. Giamarellos-Bourboulis EJ, Netea MG, Rovina N, Akinosoglou $\mathrm{K}$, Antoniadou A, Antonakos $\mathrm{N}$ et al (2020) Complex immune dysregulation in COVID-19 patients with severe respiratory failure. Cell Host Microbe 27:992-1000

6. Gust J, Hay KA, Hanafi L-A, Li D, Myerson D, Gonzalez-Cuyar LF et al (2017) Endothelial activation and blood-brain barrier disruption in neurotoxicity after adoptive immunotherapy with CD19 CAR-T cells. Cancer Discov 7:1404-1419

7. Ong EZ, Chan YFZ, Leong WY, Lee NMY, Kalimuddin S, Mohideen SMH et al (2020) A dynamic immune response shapes COVID-19 progression. Cell Host Microbe 27:879-882

8. Lucas C, Wong P, Klein J, Castro TBR, Silva J, Sundaram M et al (2020) Longitudinal analyses reveal immunological misfiring in severe COVID-19. Nature. https://doi.org/10.1038/s4158 6-020-2588-y

9. Roschewski M, Lionakis MS, Sharman JP, Roswarski J, Goy A, Monticelli MA et al (2020) Inhibition of Bruton tyrosine kinase in patients with severe COVID-19. Sci Immunol. 5:eabd0110

10. Giavridis T, van der Stegen SJC, Eyquem J, Hamieh M, Piersigilli A, Sadelain M (2018) CAR T cell-induced cytokine release syndrome is mediated by macrophages and abated by IL-1 blockade. Nat Med 24:731-738 
11. Norelli M, Camisa B, Barbiera G, Falcone L, Purevdorj A, Genua $\mathrm{M}$ et al (2018) Monocyte-derived IL-1 and IL-6 are differentially required for cytokine-release syndrome and neurotoxicity due to CAR T cells. Nat Med 24:739-748

12. Stone JH, Frigault MJ, Serling-Boyd NJ, Fernandes AD, Harvey L, Foulkes AS et al (2020) Efficacy of Tocilizumab in patients hospitalized with Covid-19. N Engl J Med 21:1-12. https://doi. org/10.1056/NEJMoa2028836

13. Ohyagi H, Onai N, Sato T, Yotsumoto S, Liu J, Akiba H et al (2013) Monocyte-derived dendritic cells perform hemophagocytosis to fine-tune excessive immune responses. Immunity 39:584-598

14. Panoskaltsis N, McCarthy NE, Knight SC (2020) Myelopoiesis of acute inflammation-lessons from TGN1412-induced cytokine storm. Cancer Immunol Immunother. https://doi.org/10.1007/ s00262-020-02702-9

15. Panoskaltsis N, McCarthy NE, Stagg AJ, Mummery CJ, Husni M, Arebi N, Greenstein D, Price CL, Al-Hassi HO, Koutinas M,
Mantalaris A, Knight SC (2020) Immune reconstitution and clinical recovery following anti-CD28 antibody (TGN1412)-induced cytokine storm. Cancer Immunol Immunother 8:1-16. https://doi. org/10.1007/s00262-020-02725-2

16. McCarthy NE, Stagg AJ, Price CL, Mann ER, Gellatly N, AlHassi HO, Knight SC, Panoskaltsis N (2020) Patients with gastrointestinal irritability after TGN1412-induced cytokine storm displayed selective expansion of gut-homing $\alpha \beta$ and $\gamma \delta$ T-cells. Cancer Immunol, Immunother 13:1-11. https://doi.org/10.1007/ s00262-020-02723-4

Publisher's Note Springer Nature remains neutral with regard to jurisdictional claims in published maps and institutional affiliations. 